\title{
THE TREATMENT OF MUSCULAR RHEUMATISM BY INJECTION.
}

\author{
$B y$ W. S. C. COPEMAN, M.D., F.R.C.P.
}

(Physician in charge of the Department of Chronic Rheumatism at the West London Hospital, and to the Hospital of St. John and St. Elizabeth)

AND

H. A. BURT, M.B., B.Ch.

(Chief Assistant, Department of Chronic Rheumatism at the West London Hospital, and at the Hospital of St. John and St. Elizabeth.)

Muscular rheumatism is probably the commonest ailment that affects mankind, yet in spite of its ubiquity little really scientific work has been done on the subject until recently, and almost nothing is known about its pathology. In the last few years certain aspects of the problem have been studied by different workers, and though the ætiology and pathology are still hidden from us, it has now definitely been established that in a large number of cases the pain of muscular rheumatism is in reality a pain referred from another structure often some distance away, rather than a lesion in the actual muscle in which pain is felt.

This is not a new conception; for a number of years it has been realised to a certain extent that referred somatic pain exists. For example most text books quote the incidence of sciatic pain arising in cases of osteo-arthritis of the hip, though in actual fact this is a rare occurrence. Many observers have recognised the incidence of sciatic pain secondary to fibrositis of the gluteus medius muscle. ${ }^{(1)}$ It can be said however that the full importance of referred pain in cases of muscular rheumatism was not appreciated until quite recently.

In 1938 papers were published in America by Steindler and Luck, and in this country by Kellgren; the former worked on the subject of low back pain, while the latter dealt with muscular pain as a whole. Steindler and Luck $^{(2)}$ found that in the 74 per cent. of their cases in which there was no arthritis, the pain down the leg was due to a ligamentous or muscular lesion in the back or pelvis. In only 26 per cent. of their cases was the pain due to actual compression of the nerve. They established their point by injecting procaine hydrochloride into the offending spot in the muscle or ligament, and considered that they had found the original site of the lesion when, and only when, the following five conditions had been satisfied:

1. Contact with the needle aggravated the local tenderness.

2. Contact with the needle elicited or aggravated the radiation down the leg.

3. Procaine infiltration suppressed the local tenderness.

4. Procaine infiltration suppressed the radiation.

5. The positive leg signs such as Lassegue's or Ober's sign had disappeared after injection.

Kellgren ${ }^{(3,4,5)}$ worked on somewhat similar lines. He first determined which place in the muscle appeared to be giving rise to the referred pain. He then chose an identically situated spot in the corresponding muscle on the opposite side of the body, and into this he injected a strong solution of saline (o.I c.c. of 6 per cent. saline). In the majority of cases this reproduced exactly the pain that the patient 
was complaining of on the affected side. He thereupon injected $\frac{1}{2}$ or I per cent. novocaine into the affected spot and the pain disappeared.

In a number of cases Kellgren found that pain was permanently relieved by a single injection; in other instances pain was relieved if the injection was repeated once or even twice at intervals of about a week. Why this should be so and how a single injection can bring about such a result can only be conjectured. Various theories have been put forward, but in fact the true reason has not been determined.

It was natural that after the publication of these papers injection of novocaine as a form of treatment was widely tried for it offered an almost magical solution to many difficulties. It is we think true to say that nearly everyone has at some time or another had magnificent results, but in many cases the results have been disappointing or at any rate not nearly so successful as the reading of Kellgren's first paper ${ }^{(3)}$ would have led us to hope for. There can be no doubt whatsoever that an injection of novocaine given in the right place to the right patient is first class treatment, but the method is not an easy one; it may be extremely difficult to determine the original site of pain. It is probably the difficulties rather than the fallacies of the method which have made some give up this form of treatment as unsatisfactory. Those who have seen Kellgren work cannot fail to be impressed by the results that he himself gets.

It appears that diffuse pain arising from a muscle always follows a segmental pattern. It will be remembered that the segmental nerve supply of the skin was worked out by $\mathrm{Head}^{(6)}$ as far back as 1893 , and further observations were made by Foerster ${ }^{(7)}$ at a later date. It is important to note that the distribution of segmental nerves to deep structures does not correspond with the segmental innervation of the skin as worked out by Head.

Two methods were employed by Kellgren in plotting out the segmental pattern of deep somatic pain. In the first method he selected a muscle which was believed to have a nerve supply from a single nerve root, as for example flexor carpi ulnaris which receives its nerve supply from the sixth cervical; into this muscle he injected strong saline and thence plotted out the area over which pain was felt.

Later a simpler and more accurate method was adopted. It was found that if saline was injected into the interspinous ligament a purely segmental area of pain and deep tenderness was produced, thus saline injected into the interspinous ligament between the sixth and seventh cervical vertebræ produced deep tenderness in the distribution of the sixth cervical segment, a pain in fact very similar to that produced by injecting flexor carpi ulnaris. By using these methods the whole of the body was mapped out into areas from the first cervical segment to the second sacral. For full details Kellgren's original papers must be consulted, but roughly speaking the distribution is as follows: In the upper limb cervical 5 is represented over the deltoid region, cervical 6 along the outside of the arm and forearm, cervical 7 over the back of the arm and forearm, cervical 8 along the inner aspect of the forearm and the first thoracic segment supplies the deep structures on the inner aspect of the arm. In the lower limb lumbar 4 and 5 are referred to the outer side of the thigh and leg and also over the front of the thigh over and just above the knee, while sacral I and 2 are represented over the back of the thigh and leg. 
The cases which are suitable for the injection of novocaine arrange themselves under four main headings :

1. Where the pain is due to a recent injury or strain in the muscles or ligaments.

2. In certain cases of long standing pain, where injury or strain is probably the cause, though in many instances not so obvious a cause as in the type described above.

3. In a few cases of diffuse muscular pain, as for example in generalised fibrositis of the gluteus maximus where the inflammatory process appears likely to involve the underlying sciatic nerve.

4. As a diagnostic measure to detect the source of acute or chronic muscular pain.

\section{Injection Treatment for Acute Pain of Recent Onset.}

The first type of case mentioned above, namely the acute traumatic type is probably the most satisfactory and certainly the easiest with which to deal. Patient's histories are notoriously inaccurate, and when it comes to their locating the site of an injury which occurred some time ago and which is no longer acutely painful, accuracy becomes impossible. For this reason if a patient is seen shortly after a sudden twist or strain an accurate localisation of the lesion is much more easily obtainable. In the first few hours, it is true, there is commonly considerable spasm of the surrounding muscle, and where a-ligament is involved the overlying muscle is held in spasm also. After a period of rest, sometimes assisted by strapping, sometimes by heat, the spasm is relieved and the exact site of the lesion can be located. Into this place .5 per cent. novocaine may be injected ; the exact amount varies, but when the right spot is reached by the needle between three and five c.cs. are usually sufficient.

Examples of this type suitable for injection are those of lumbar pain coming on suddenly during or shortly after a spell of digging in the garden. This is particularly liable to happen to one not used to strenuous gardening. At the present moment therefore lumbago arising in this way is particularly common. Acute sciatic pain of. sudden onset does not respond satisfactorily to injection therapy for the majority of cases where the onset occurs during exercise are due to sacro iliac strain, a condition which responds much better to manipulation. The sudden onset of pain in the arm or shoulder is frequently muscular in origin, and therefore this type of case is also suitable for novocaine injection.

\section{Injection Treatment in cases of Chronic Muscular Pain.}

According to Kellgren many cases of chronic muscular pain of long standing (he himself quotes cases with six and seven months' history) respond remarkably well to novocaine injection. These cases are often extraordinarily difficult to treat, and it is now becoming clearer why this should be so. As stated above a lesion in a muscle supplied by a certain segmental nerve may give rise to pain in any part of the body supplied by that nerve. If a muscle is supplied by more than one nerve it follows that pain can be felt over a considerable area. A particular case illustrates this point:

The patient was a woman of twenty-eight who spent her day peeling vegetables in an Oxford Street store. She came up to the out-patient department complaining of pain in a number of situations, namely the right side of the neck (C 3 and 4), the outside of her right arm (C 5), the upper and outer side of her forearm and the thenar eminence 
(C 6). There was no history of injury but one suspected that the condition had something to do with her work and was, in fact, traumatic in origin. The site of the pain did not fit in with any particular nerve lesion, nor were there any signs of organic nervous disease. On examination each place that the patient said was painful was found to be tender on palpation, but this tenderness was felt only at the site of palpation and did not give rise to pain in the other tender sites. When the muscles of the shoulder girdle were carefully examined a tender spot was found in the substance of the pectoralis major, and when this was palpated the patient complained of a dull ache in all the sites, namely the trapezius, the deltoid, the supinator and the thenar eminence. The pectoralis major has a complicated nerve supply owing to the fact that it is intimately connected with the overlying fascia. The muscle itself is supplied by the sixth, seventh and eighth cervical segments, the fascia has an extensive nerve supply which includes the third and fourth cervical as well as the upper dorsal segments. It is quite understand. able that a lesion in the pectoralis major can give rise to pain over a large area and sometimes over a number of apparently unconnected areas. This particular patient immediately responded to 15 c.cs. of .5 per cent. novocaine; three days later the pain returned to a less degree but a second injection in the same situation produced a permanent cure.

In dealing with a case like that just described it is necessary first to have some idea of the segmental distribution of referred somatic pain. In the second place valuable information can often be obtained by discovering what particular action or movement brings on the pain. One patient for example complained that she got pain after she had been polishing taps for a few minutes. It was not clear exactly where the lesion was situated so the patient was asked to polish the taps in the out-patient department until the pain came on. It was then quite an easy matter to locate the painful spot and the patient obtained permanent relief after injection.

Another way in which an obscure spot can be located is to enlist the help of a masseur and to get him to massage the areas which are likely to contain the source of the pain. Frequently the site is masked by spasm of the whole muscle and as a result of massage the spasm is overcome and the offending spot can be unmasked.

Reference has already been made to the paper by Steindler and Luck in which they laid down their five conditions, all of which had to be satisfied before they considered that the lesion had been located. In many cases it appears that the injection has been successful, the pain disappears and palpation of the tender area produces no symptoms, yet in a few hours all the pain returns. In other cases the pain disappears never to return. The question arises whether there is any way of knowing at the time of injection whether the right place has really been attacked. It appears to us that when the correct place has been located not only is the pain produced immediately the needle is inserted, but it is relieved immediately the novocaine is injected. If there is a latent period between the injection of novocaine and the relief of pain, or if the pain is only partly relieved it is likely that the correct place has not been found. Often information can be obtained from the patient's own words. In a partially successful case the patient may say, "That is better, the pain seems to be going off," whereas in a really successful case he will exclaim, "Why, the pain has gone!"

It is not at all unusual for a certain amount of aching to be felt a few hours after the injection has been given (presumably when the effect of the local anæsthetic has worn off). This pain may last twenty-four and even thirty-six hours in a case which ultimately turns out to be a complete success. It is always wise to warn the patient that a period of aching is likely to follow the injection. 
The needles to be used should be of fine bore, a diameter intermediate between a hypodermic and an intravenous needle is the best. If the needle is too narrow it will be difficult to get through ligamentous structures; if it is too wide damage may be done to the muscles. If the needles are kept in spirit it is most important to see that they are well washed out with sterile water or saline for if even a small amount of spirit finds its way into muscle a very painful condition may arise.

Certain situations are particularly suitable for injection. As in the acute traumatic group these include the muscles of the shoulder girdle and the lumbar region, and to them may also be added muscles distal to the elbow joint. With regard to the shoulder, a large number of cases of so called brachial neuritis are undoubtedly examples of pain referred from the muscles arising from the scapula or ribs. An example of this has already been given in some detail and other similar instances could be cited. The cases of brachial neuritis secondary to changes in the sub-acromial bursa do not respond to injection therapy, and where the condition is chronic and the wall of the bursa definitely thickened surgical interference may be necessary.

In the condition popularly known as "tenmis elbow" it is usually possible to find a tender spot in the supinator muscle, frequently near its periosteal attachment ; less commonly there is a lesion in the pronator teres. A small injection of novocaine may be highly successful. The effect is sometimes increased if the patient is made to go through full movements of pronation and supination after the injection.

There are few occasions on which injection treatment can be used in diffuse muscular pain. The example of fibrositis of the gluteus maximus has been cited, and it has been claimed by some that well timed injection has prevented the spread of inflammation from the muscle to the underlying sciatic nerve. Various substances have been used in this connection including oxygen, .5 or I.o per cent. novocaine, and normal saline. Oxygen is not employed by many workers, and does not seem to have any advantage over the other substances. In dealing with a large area of muscle a considerable quantity of solution may have to be injected; as novocaine in quantity may, however, have an unpleasant effect on the patient it is usual to use novocaine for infiltration of the skin and normal saline for the rest of the injection.

In cases of sciatic pain arising secondary to fibrositis of the gluteus maximus it was at one time advocated that the nerve itself should be injected. This, most people now agree, is unwise; the injection should be made around the sciatic nerve and not into its substance. The amount injected may be $50 \mathrm{c.cs}$. and much larger quantities may be given if normal saline is used. Sometimes very definite relief is given by this method of treatment.

\section{Injection of novocaine as a diagnostic measure.}

The use of novocaine in this connection has already been mentioned in the earlier part of this paper. Its particular value is in those cases in which the question of surgery arises. Most of the lesions of the interspinous ligaments, and many instances of shortening or fibrosis of the tensor fascia lata respond most disappointingly to physio-therapy and only very temporarily to injection treatment; in these cases surgery may afford the only chance of relief. It is well worth while to try the effect of an injection of novocaine on such a patient. If all the symptoms and signs disappear entirely, howbeit temporarily, as a result of the injection, it is likely first that the correct site of the lesion has been located, and secondly, that operative interference in this site will bring about permanent relief. 
Substances used for injection.

The substances that we have used for the most part have been aqueous solution of novocaine (.5 or I.o per cent.) and normal saline. We have tried the oily preparations of novocaine, but the effect does not seem to have been in any way better than the aqueous solution. It has the disadvantage of requiring a bigger needle, which is more painful for the patient on the one hand, and more liable to damage the muscle on the other.

\section{Summary.}

I. An account is given of the experimental work which led up to the employment of novocaine injection in the treatment of muscular rheumatism.

2. The cases which are suitable for injection are discussed and examples of patients treated are given. There are four main groups, the acute muscular or ligamentous group which is usually traumatic in origin, the chronic group which also may be traumatic, the group where there is diffuse muscular involvement and, finally, the group where it is important to find the exact site of the lesion with a possible view of subsequent surgery.

3. The methods of injection are described and an attempt is made to account for the failures and pitfalls of the method.

4. The relative merits of the substances used for injection are discussed.

REFERENCEs.

(1) BURT, Proc. Roy. Soc. Med. Vol. 28. p.77.

(2) STEINDLER and LUCK, J.A.M.A. 110, 2 .

KELLGREN, B.M.J. 1938, 1, 325.

KELLGREN Clinical Science $1938,3,175$.

KELLGREN, ibid 1939, 4, 34 .

HEAD, Brain. 1893 16, 1.

FOERSTER, Brain. 1933, 56, 1.

(8) MENNELL, "Backache." London 1931

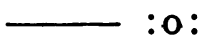

